\title{
Leukemia cutis with IDH1, DNMT3A and NRAS mutations conferring resistance to venetoclax plus 5 -azacytidine in refractory AML
}

JingHan Wang ${ }^{1,2+}$, Xingnong Ye $e^{1,2,3+}$, Cuihua Fan ${ }^{4 \dagger}$, Jie Zhou ${ }^{3}$, Shuna Luo ${ }^{3}$, Jingxia Jin $^{3}$, Dan Chen ${ }^{3}$, Yan Zheng ${ }^{3}$, Cai $\mathrm{Wu}^{3}$, Xiaoqiong $\mathrm{Zhu}^{3}$, Jie $\mathrm{Jin}^{1,2,3^{*}}$ (D) and Jian Huang ${ }^{1,2,3^{*}}$

\begin{abstract}
r
Recently, novel drugs like venetoclax plus 5-azacytidine (VA) were reported to have promising efficacy in refractory acute myeloid leukemia (AML). However, there are still some cases presented with novel drugs resistance, and its genetics composition and clinical phenotype are urging to study. We described a 58-year-old patient who was resistant to intensive chemotherapy. This refractory AML was presented with the persistence of RUNXI, IDH1 and DNMT3A mutations. RUNX1 mutations disappeared and leukemia cutis ensued after multiple chemotherapies. Leukemia cutis exhibited NRAS mutations in addition to IDHI and DNMT3A mutations. With the VA salvage treatment, platelets were recovered to the normal level and blasts in bone marrow and peripheral blood were moderately controlled. However, leukemia cutis did not resolve. Unexpectedly, BM blasts obtained the new NRAS mutations after VA treatment, and consequently experienced leukostasis with two distinct leukemia clones. After survival of 230 days, this patient died because of spontaneous cerebral hemorrhage. This case highlights presentation of leukemia cutis with simultaneous mutations of IDHI, DNMT3A and NRAS in AML patients might act as a resistant niche to avoid the toxicity of multiple drugs including VA. There is unmet need to validate this result in the clinical trials or a large cohort of patients in the future.
\end{abstract}

Keywords: Acute myeloid leukemia, BCL-2 inhibitors, Leukemia cutis

\section{To the Editor:}

Approximately $30 \%$ of newly diagnosed patients with acute myeloid leukemia (AML) do not achieve complete remission with intensive induction therapy, and therefore are classified as refractory or resistant disease (RRD) [1]. RRD is among the most challenging scenarios in AML management. With the

\footnotetext{
* Correspondence: jiej0503@zju.edu.cn; househuang@zju.edu.cn

${ }^{\dagger}$ Jing Han Wang, Xingnong Ye and Cuihua Fan contributed equally to this work.

'Department of Hematology, The First Affiliated Hospital, Zhejiang University College of Medicine, Hangzhou, P.R. China

Full list of author information is available at the end of the article
}

growing clinical translation of genomics into daily routine [2-5], RRD has been becoming an important field for novel drug investigation. Recently, the welltolerated regimens venetoclax plus 5-azacytidine (VA) were proved to be highly effective in these patients [6]. However, the features related to VA resistance are still under investigation. Here, we present with a RRD patient with a clinical and molecular picture of VA resistance.

The patient is a 58-year-old man with a morphological and immunological diagnosis of AML-M2 (Fig. 1a-b) and a past history of myocardial infarction (MI). His physical examination was 


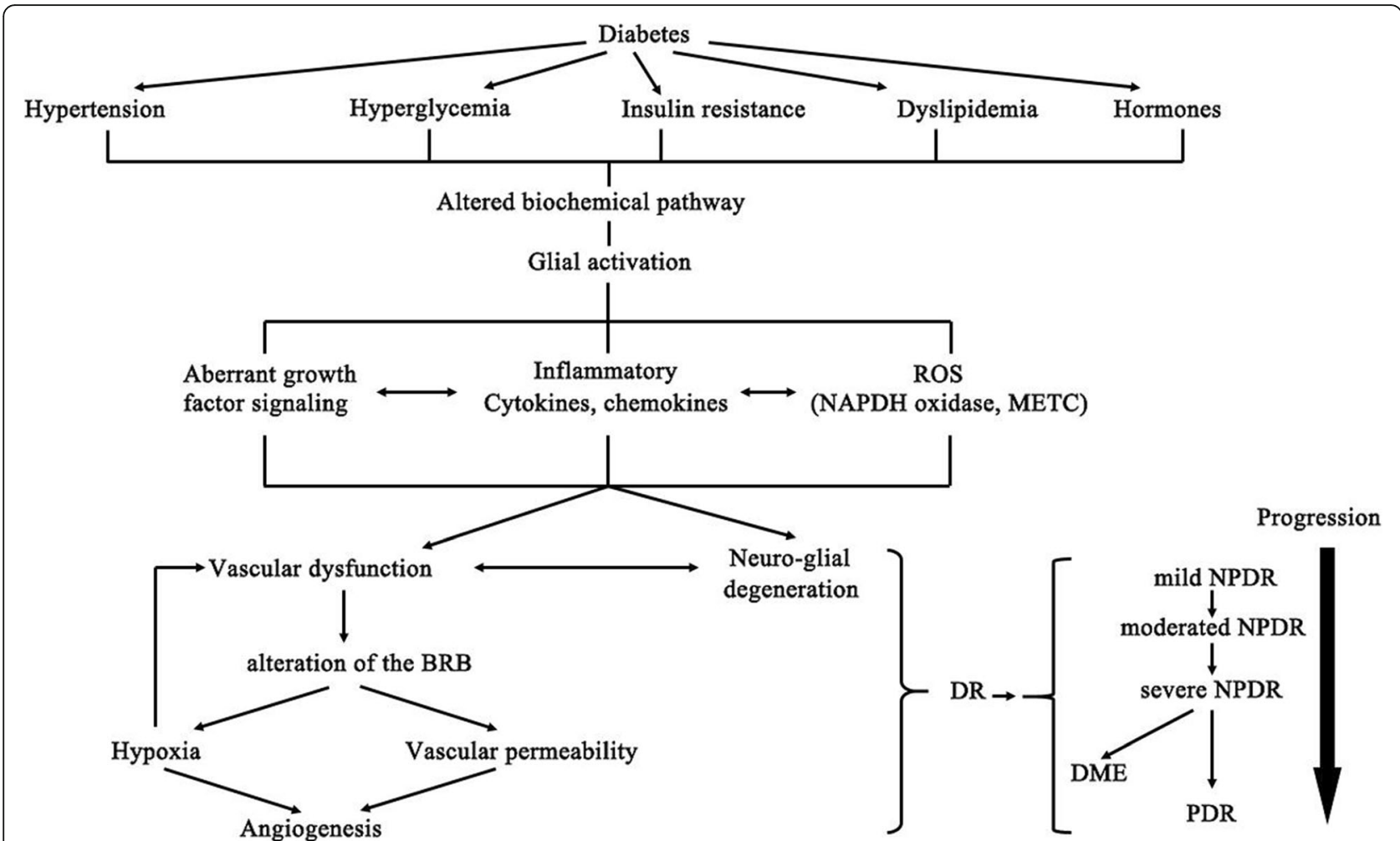

Fig. 1 Clinical, molecular and histological features of this refractory AML patients.(A) The morphology of bone marrow (BM) blasts $(\times 1000)$. (B) The immunophenotype of BM blasts at diagnosis. Red colors in J region represent BM blasts. Flow cytometry determines $45 \%$ blasts and demonstrates MPO, CD38 and HLA-DR dim expression, CD33, CD117, CD13, CD34, CD9 and CD123 positivity, while CD2, CD5, CD7, CD10, CD19, CD79a, CD11b negativity. (C) The immunophenotype of peripheral blood cells when leukemocytosis ensues. Red and blue colors illustrate two leukemia clones. (D) Leukemia cutis in this patient. (E) The morphology analysis in skin biopsy sample (H\&E × 100). (F) The immunohistochemistry displays MPO positive in skin biopsy sample $(\times 100)$. (G) The immunohistochemistry displays CD15 positive in skin biopsy sample $(\times 100)$. $(H)$ The immunohistochemistry displays BCL-2 positive in blasts of the bone marrow biopsy $(\times 100)$. (I) The immunohistochemistry displays a morsol of $\mathrm{BCL}-2$ positive in skin biopsy sample $(\times 100)$

unremarkable. At the time of diagnosis, the percentage of blasts was $66 \%$, and the karyotype was normal (Figure S1). A peripheral blood test was notable for a substandently leukocytosis with WBC $104 \times 10^{\wedge} 9 / \mathrm{L}$, hemoglobin $104 \mathrm{~g} / \mathrm{L}$, and platelets 60 $\times 10^{\wedge} / \mathrm{L}$. As shown in treatment flowchart (Figure S2), induction chemotherapy with HAA based regimens (homoharringtonin $2 \mathrm{mg} / \mathrm{m} 2$ daily for 7 days, cytarabine $100 \mathrm{mg} / \mathrm{m} 2$ daily for 7 days, aclarubicin $20 \mathrm{mg}$ daily for 7 days) was started [7], but bone marrow (BM) blasts reached $9 \%$ on day 15 and surged up to $36 \%$ on day 30 indicating poor response (Figure S3). NGS analyses had revealed IDH1 (exon4:c.394C > G:p.Arg132Gly), DNMT3A (exon19:c.2078G > A:p.Arg693His) and RUNX1(exon1:c.86 T > C:p.Leu29Ser) mutations (Fig. 2 and Table S1). Based on genetic results, decitabine plus standard IA regimen (decitabine $20 \mathrm{mg} / \mathrm{m} 2$, days $1-5$; idarubicin $10 \mathrm{mg} / \mathrm{m} 2$ daily for 3 days and cytarabine $100 \mathrm{mg} / \mathrm{m} 2$ daily for 7 days) were used as the re-induction therapy. About 1 month later, bone marrow smear revealed a morphological complete remission (CR) with $3 \%$ blasts, while platelet was not recovered (Figure S3B). Thus, CR with incomplete platelet recovery was rendered. Therefore, treatment with decitabine plus IA was immediatetly initiated as a bridge to allogeneic hematopoietic stem cell transplantation. Unfortunately, he began to note skin lesion, although BM blasts were stable for approximately 2 months. After treatment of 130 days, leukemia cells increased up to $20 \%$ in the peripheral blood and $6 \%$ in bone marrow with a normal karyotype (Figure S1). Physical examination showed numerous dermal gray-blue papules (Fig. 1d). No evidence of leukemia blasts involvement was observed in the Computed Tomography lung screening, hepatic ultrasound, and cerebral Magnetic Resonance Imaging, respectively (Figure S4). Biopsy of the skin lesion demonstrated a dermal infiltration of myeloblast population, which was illustrated by diffuse reactivity for CD15 and MPO immunostains (Fig. 


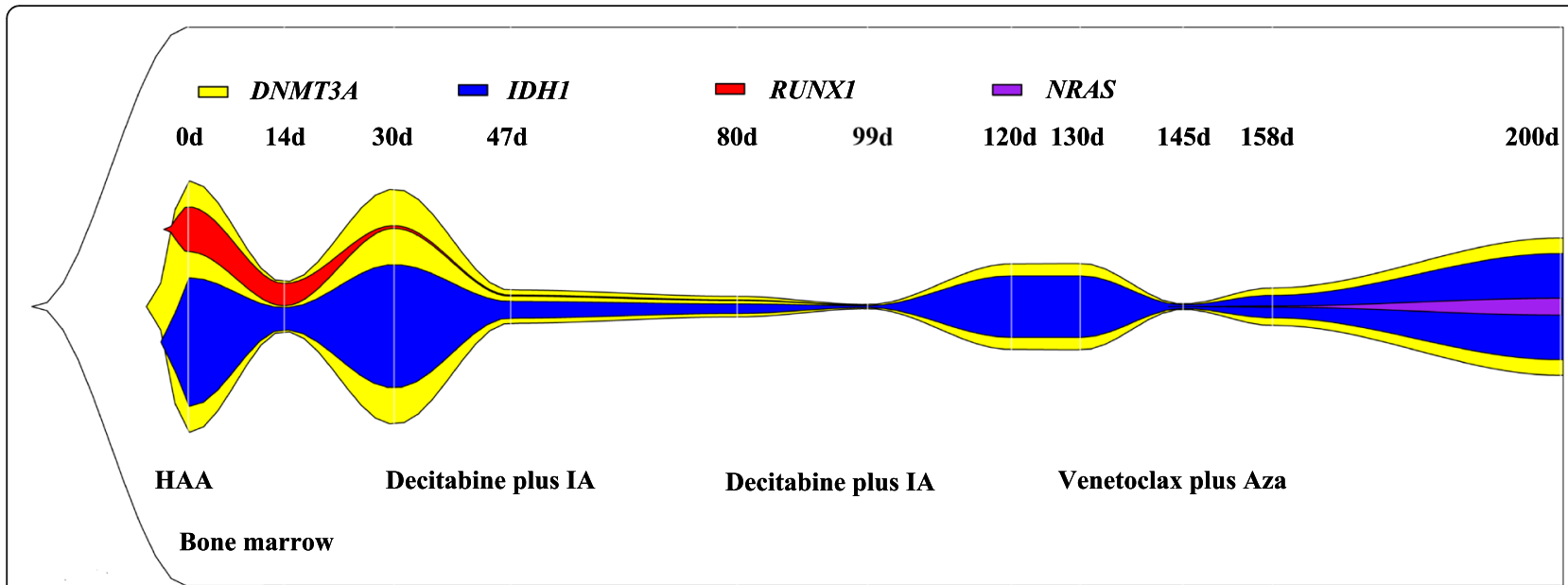

Fig. 2 The clone progression following mulitple chemodrugs and venetoclax treatment. Schematic of a possible model clone evolution inferred from next-generation sequencing data combined with the percentage of blasts detected by flow cytometry and visualized using fishplot $\mathrm{R}$ packages [11]. Here, we found the variate allele frequence (VAF) of DNMT3A is higher than that of IDHI mutation at daignosis, implying IDHI mutations occurring later than DNMT3A mutations. Following the intensive chemotherapy, RUNX1 mutated clone disappeared, but IDH1 and DNMT3A mutated clones still survived. Notably, the leukeiam cutaneous with additional NRAS mutations did not resolve during VA regimens. Thus, these clones in skin can persist over the time of the intensive chemotherapy and obtain resistance to targeted therapy, leading to further clonal expansion and eventually causing recurrent disease in the blood and bone marrow

1e-f-g). Notably, NGS demonstrated NRAS (exon2: c.38G > A:p.Gly13Asp), DNMT3A and IDH1 (Table S1) mutations coexisting in leukemia cutis, but RUNX1 negativity and DNMT3A and IDH1 positivity exhibiting in refractory $B M$ samples, which was distingusished with the initially mutated pattern of BM blasts. Putting the leukemia cutis and the chemodrug resistant blasts together, AML refractory disease was definitely diagnosed. As DiNardo et al. reported using ivosidenib (an inhibitor of mutant IDH1) to treat IDH1-mutated relapsed or refractory AMLs, the median durations of responses were more than 8 months with $30.4 \% \mathrm{CR}$ rate [8]. The major side effects were differentiation syndrome and prolongnation of QT interval. Based on these studies, this patient might not fit for ivosidenib treatment due to the MI history. By contrast, another novel drug venetoclax was also sensitive in IDH1 mutant primary AML cells with less drug toxicity $[9,10]$. Thus, we treated this patient with venetoclax combining with 5 -azacytidine (venetoclax $400 \mathrm{mg}$ and intravenous azacitidine $75 \mathrm{mg} / \mathrm{m}^{2}$ [days 1-7 of each 28-day cycle]) [6]. At venetoclax initiation, despite $\mathrm{WBC}$ up to $20 \times 10^{\wedge} 9 / \mathrm{L}$, we did not observe tumor lysis syndrome. With this regimen, platelets were recovered to the normal level and blasts (4.5 and 6.7\% respectively in bone marrow and peripheral blood) were moderately controlled. However, the patient's skin lesion did not resolve during the course of VA treatment. At the survival time of 200 days, WBC increased rapidly up to more than $50 \times 10^{\wedge} 9 / \mathrm{L}$ and the immunophenotypic data revealed two clonal architecture of neoplasia in the peripheral blood (Fig. 1c). In addition, NGS diagnosis showed the same as its initial mutated genes of $I D H 1$ and DNMT3A were still positive and unexpectedly NRAS mutation in blood was incurred after 1 months of VA treatment (Fig. 2 and Table S1). We hypothezed the action of myeloid leukemia clones transferring to skin tissue as a resistant niche to avoid the toxicity of venetoclax. Therefore, we measured the expression of BCL-2 of both BM biopsy (Fig. 1h) and cutaneous blasts (Fig. 1i). As a result, the more and higher expression of BCL-2 was observed in BM biopsy than in leukemia cutis. After survival of 230 days, this patient unfortunately died because of spontaneous cerebral hemorrhage.

\section{Supplementary Information}

The online version contains supplementary material available at https://doi. org/10.1186/s40364-020-00246-9.

Additional file 1 Figure S1. Normal karyotype was illustrated at the time of AML diagnosis (A), morphological complete remission (B), and leukemia refractory and resistance $(C)$, respectively. Figure S2. Treatment flowchart illustrates in this study. Figure S3. The morphology of bone marrow $(B M)$ blasts $(\times 1000)$ indicated no complete remission on day 30 after HAA treatment(A), a morphological complete remission after treatment with decitabine plus $I A(B)$, leukemia relapse after the second course of decitabine plus $I A(C)$, and moderately control under VA treatment. Figure S4. No evidence of leukemia blasts involvement was observed in the Computed Tomography lung screening (A), hepatic 
ultrasound(B), and cerebral Magnetic Resonance Imaging(C), respectively. Table S1. The detailed information of gene mutations.

\section{Abbreviations}

VA: Venetoclax plus 5-azacytidine; AML: Acute myeloid leukemia; BM: Bone marrow

\section{Acknowledgements}

We thank the patient for permission to present his case.

\section{Availiability of data and materials}

The data analyzed was seen in this text and supplementary data.

\section{Authors' contributions}

J.H.W, J.J. and J. H planned the treatment, J.H.W wrote the manuscript, C.H.F, S. L, J.X.J, D. C, Y. Z, C. W, X.Q.Z followed the patient. Y. Z, C. W and J. Z were responsible for histopathology and flow cytometry, J.H.W, J. J and J. H revealed the mutations; and all authors critically read and approved the draft manuscript.

\section{Funding}

This work is supported by Zhejiang Provincial Natural Science Foundation of China (LY19H080009); Zhejiang Public Technology Application Research Program, China (LGF21H080003); and the Key Project of Jinhua Science and Technology Plan, China (2020XG-29 and 2020-3-011). The funders had no role in study design, data collection, data analysis, interpretation, writing of this report.

\section{Ethics approval and consent to participate}

The study was approved by the Institutional Review Board of the Fourth Affiliated Hospital of Zhejiang University. This patient was informed about the study and gave an informed consent.

\section{Consent for publication}

Consent for publication was provided by this patient.

\section{Competing interests}

The authors declare that they have no competing interests.

\section{Author details}

${ }^{1}$ Department of Hematology, The First Affiliated Hospital, Zhejiang University College of Medicine, Hangzhou, P.R. China. ${ }^{2}$ Key Laboratory of Hematologic Malignancies, Diagnosis and Treatment, Zhejiang, Hangzhou, PR China. ${ }^{3}$ Department of Hematology of the Fourth Affiliated Hospital Zhejiang University School of Medicine, Yiwu, P.R. China. ${ }^{4}$ Department of Hematology, Shulan Hospital, Hangzhou, P.R. China.

Received: 24 September 2020 Accepted: 5 November 2020

Published online: 25 November 2020

\section{References}

1. Thol F, Schlenk RF, Heuser M, Ganser A. How I treat refractory and early relapsed acute myeloid leukemia. Blood. 2015;126(3):319-27.

2. Schnittger S, Dicker F, Kern W, Wendland N, Sundermann J, Alpermann T, Haferlach C, Haferlach T. RUNX1 mutations are frequent in de novo AML with noncomplex karyotype and confer an unfavorable prognosis. Blood. 2011:117(8):2348-57.

3. Bullinger L, Dohner K, Dohner H. Genomics of acute myeloid leukemia diagnosis and pathways. J Clin Oncol. 2017:35(9):934-46.

4. Chan SM, Majeti R. Role of DNMT3A, TET2, and IDH1/2 mutations in preleukemic stem cells in acute myeloid leukemia. Int J Hematol. 2013;98(6): 648-57.

5. Dohner H, Estey E, Grimwade D, Amadori S, Appelbaum FR, Buchner T, Dombret H, Ebert BL, Fenaux P, Larson RA, et al. Diagnosis and management of AML in adults: 2017 ELN recommendations from an international expert panel. Blood. 2017:129(4):424-47.

6. DiNardo CD, Pratz KW, Letai A, Jonas BA, Wei AH, Thirman M, Arellano M, Frattini MG, Kantarjian H, Popovic R, et al. Safety and preliminary efficacy of venetoclax with decitabine or azacitidine in elderly patients with previously untreated acute myeloid leukaemia: a non-randomised, open-label, phase 1b study. Lancet Oncol. 2018;19(2):216-28.

7. Jin J, Wang JX, Chen FF, Wu DP, Hu J, Zhou JF, Hu JD, Wang JM, Li JY, Huang $X J$, et al. Homoharringtonine-based induction regimens for patients with de-novo acute myeloid leukaemia: a multicentre, open-label, randomised, controlled phase 3 trial. Lancet Oncol. 2013;14(7):599-608.

8. DiNardo CD, Stein EM, de Botton S, Roboz GJ, Altman JK, Mims AS, Swords $\mathrm{R}$, Collins RH, Mannis GN, Pollyea DA, et al. Durable remissions with ivosidenib in IDH1-mutated relapsed or refractory AML. N Engl J Med. 2018; 378(25):2386-98.

9. Konopleva M, Pollyea DA, Potluri J, Chyla B, Hogdal L, Busman T, McKeegan E, Salem AH, Zhu M, Ricker JL, et al. Efficacy and biological correlates of response in a phase II study of venetoclax monotherapy in patients with acute myelogenous leukemia. Cancer Disc. 2016;6(10):1106-17.

10. Chan SM, Thomas D, Corces-Zimmerman MR, Xavy S, Rastogi S, Hong WJ, Zhao F, Medeiros BC, Tyvoll DA, Majeti R. Isocitrate dehydrogenase 1 and 2 mutations induce $\mathrm{BCL}-2$ dependence in acute myeloid leukemia. Nat Med. 2015;21(2):178-84

11. Miller CA, McMichael J, Dang HX, Maher CA, Ding L, Ley TJ, Mardis ER, Wilson RK. Visualizing tumor evolution with the fishplot package for R. BMC Genomics. 2016;17(1):880.

\section{Publisher's Note}

Springer Nature remains neutral with regard to jurisdictional claims in published maps and institutional affiliations.
Ready to submit your research? Choose BMC and benefit from:

- fast, convenient online submission

- thorough peer review by experienced researchers in your field

- rapid publication on acceptance

- support for research data, including large and complex data types

- gold Open Access which fosters wider collaboration and increased citations

- maximum visibility for your research: over $100 \mathrm{M}$ website views per year

At $\mathrm{BMC}$, research is always in progress.

Learn more biomedcentral.com/submissions 